\title{
Terahertz gyrotrons with quasi-regular cavities
}

\author{
I.V. Osharin, I.V. Bandurkin, Yu.K. Kalynov, S.V. Kuzikov, A.V. Savilov
}

Institute of Applied Physics, Nizhny Novgorod, 603950, Russian Federation, osharin@appl.sci-nnov.ru

The gyrotron $[1,2]$ is widespread variety of electron cyclotron masers based on the selective excitation of a near-cutoff mode of an open microwave cavity by a beam of electrons rotation in a magnetostatic field. The principal advantages of the gyrotron are high mode selectivity achieved in simple microwave systems and weak sensitivity of the electron-wave interaction to velocity spread of electrons. Since the output frequency of the gyrotron is close to the electron cyclotron frequency or its harmonics, a natural way to decrease the operating magnetic field is the use of operation at the harmonics of the cyclotron frequency. However, at a fixed electron current, a longer operating cavity is required, in general, to provide the start of oscillations at a high cyclotron harmonic. In this situation, the start of the parasitic oscillations of lowfrequency waves excited at lower cyclotron harmonics is dangerous.

Thus, a careful mode selection and suppression of the parasitic modes have to be ensured in a gyrotron operating at a high cyclotron harmonic. In [4], a method of selective discrimination of parasitic low-frequency modes in a second cyclotron harmonic gyrotron was proposed, based on the use of special phase correctors located inside the gyrotron resonator. The selection principle is based on the fact that the corrector provides different phase shifts for quasi-critical waves at the fundamental and operating cyclotron harmonics. The method is based on the singlemode approximation, when the corrector is represented by a relatively small and smooth widening of the cavity wall, and the wave phase shift can be calculated in the WKB approximation. Such an approach can be used in sufficiently long and low-mode resonators. However, in the case of a short cavity operating at a high radial mode, this approach can not be realized. This is due to the fact that the restriction on the maximum length of the corrector imposes a restriction on its minimum depth. From a certain set of the resonator parameters, the single-mode approximation in describing the corrector becomes invalid $[5,6]$.

We propose a modification of the described method of low-frequency parasitic modes discrimination in a gyrotron at the second cyclotron harmonic, which makes it possible to use in relatively short and high-mode resonators. The ultimate goal of the proposed method, as well as in [4], is to realize inside the gyrotron cavity of an element that would noticeably distort the field of parasitic low-frequency modes but would not significantly affect the field of the operating mode at the second cyclotron harmonic. Under certain conditions, a rectangular groove in the resonator wall (Fig. 1) can serve as such an element. Namely, if the radius of the resonator inside this groove is close to the critical one for a mode whose radial index exceeds by one the radial index of the operating mode, and whose azimuthal index coincides with one of the operating mode, then by choosing the width of the groove, it is possible to achieve almost complete absence of scattering of the operating mode on this element. a)

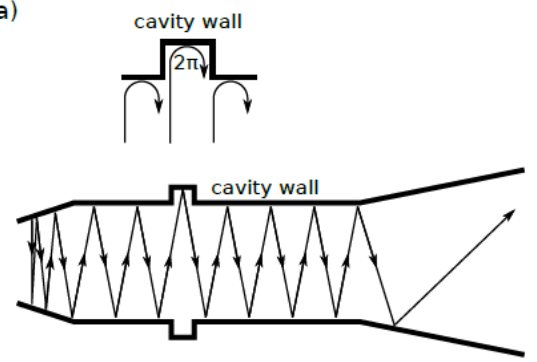

b)

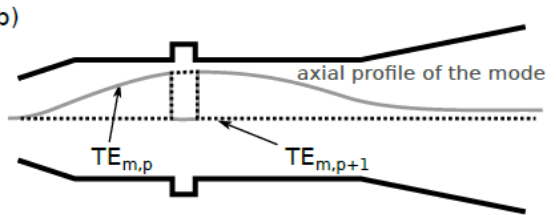

c)

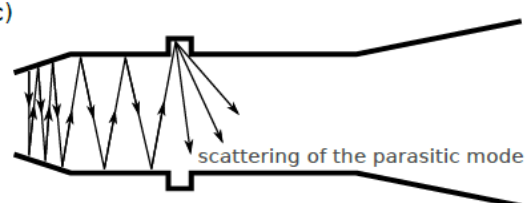

Fig. 1. Explanation of the selection mechanism from the Brillouin concept (a) and cavity with modes transformation point of view (b), as well as illustration of low harmonic mode scattering (c)

To demonstrate the method, we propose to modify the cavity of a $670 \mathrm{GHz}$ gyrotron which presently operates in the Institute of applied physics (IAP) at the fundamental cyclotron resonance at the TE31,8 mode [3]. An analysis of the eigenmodes spectrum of this cavity shows that in the operating range of magnetic fields, the resonance is possible at the second cyclotron harmonic at the TE63,15 mode at $1.34 \mathrm{THz}$. Nevertheless, in the traditional cylindrical system the starting current of this mode is slightly higher than the starting current of the lowfrequency modes TE30,8 and TE31,8 excited at the fundamental cyclotron resonance (Fig. 2).

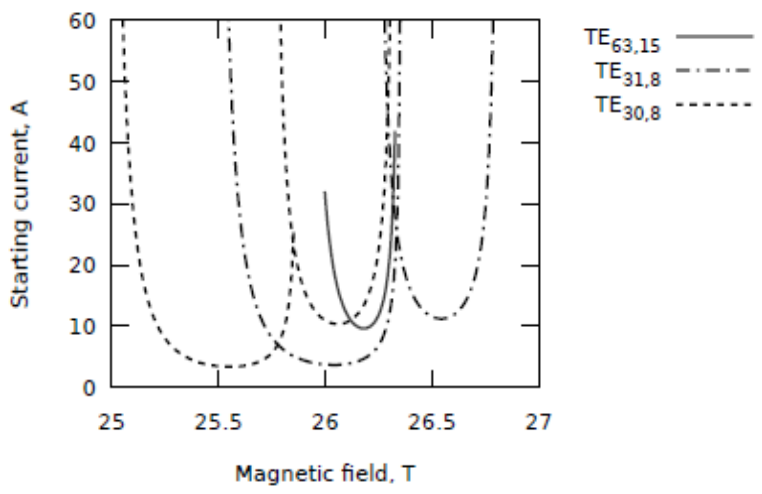

Fig. 2. Starting currents of the fundamental and second cyclotron harmonic oscillations in the regular $670 \mathrm{GHz}$ gyrotron cavity 
To discriminate these modes, we propose to apply a groove with depth of about $0.12 \mathrm{~mm}$ to the cavity wall having a radius of $4.54 \mathrm{~mm}$. With this depth, the radius of the resonator inside the groove approximately corresponds to the critical radius for the TE63,16 mode. The longitudinal position of the groove along the axis of the resonator should divide its length in the ratio 1 to 3 (Fig. 3), which makes it possible to discriminate not only the basic parasitic axial modes, but also modes with two variations of the field along the axis. The eigenmodes of the modified resonator were calculated by solving the threedimensional Maxwell equations with a given field dependence on the azimuthal coordinate corresponding to the azimuthal index of the mode. The simulation was carried out by the finite element method in the two-dimensional domain (r-z) [5].

a)

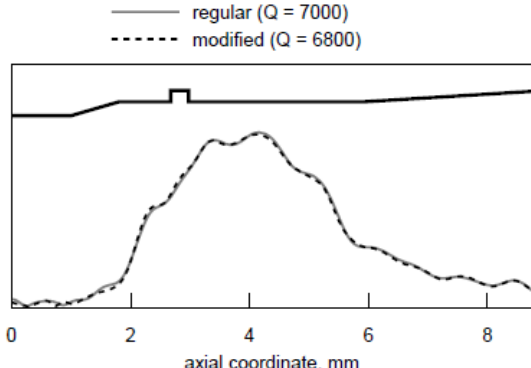

b)

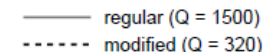

.... modified $(Q=320)$

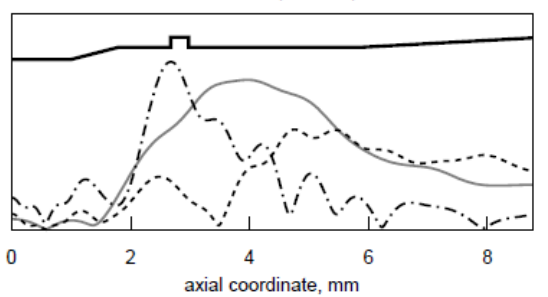

c)

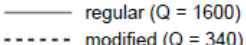
-. - . $-\operatorname{modified}(Q=450)$

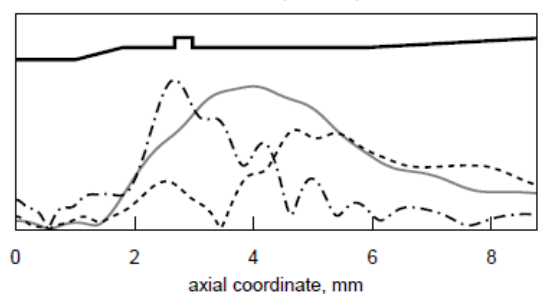

Fig. 3. Results of electrodynamic cavity simulations. Comparison of the axial profiles of the TE63,15 (a), TE30,8 (b) and TE31,8 (c) modes in the vicinity of electron beam for the regular and modified cavity. In the modified cavity, two modes with approximately equal low Q-factors appear in last two cases, which are actually the descendants of the two lowest axial modes of the regular cavity.

By choosing the geometric dimensions of the groove it was possible to achieve its almost complete "invisibility" for the TE63,15 mode, keeping both the quality factor of this mode and the longitudinal field profile in the position of the electron beam unchanged (Fig. 3a). Calculation of the eigenmodes of the modified resonator near the resonance at the first cyclotron harmonic, as ex- pected, demonstrated a strong distortion of the longitudinal profiles of these modes with respect to the unperturbed system and a significant decrease in their Qfactors (Fig. 3b,c). Calculation of the starting currents confirmed the effectiveness of discrimination of parasitic modes in the proposed resonator (Fig. 4). The deformation of the low-frequency modes turned out to be so strong that their starting currents became several times greater than the practically unchanged starting current of the operating mode at the second cyclotron harmonic.

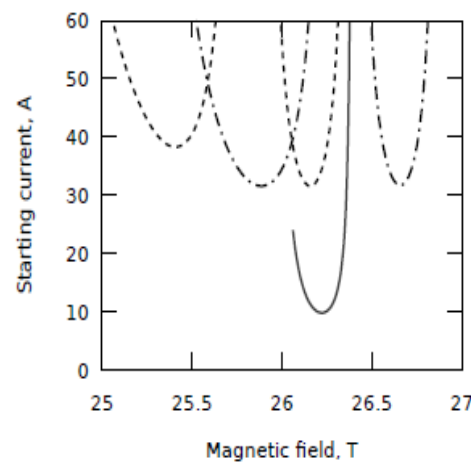

$$
\begin{aligned}
& \mathrm{TE}_{63,15}- \\
& \mathrm{TE}_{31,8}-\cdot \cdot \cdot \cdot \\
& \mathrm{TE}_{30,8} \cdot \cdots \cdot
\end{aligned}
$$

Fig. 4. Starting currents of the fundamental and second cyclotron harmonic oscillations in the modified gyrotron cavity

The proposed method of discrimination of parasitic low-frequency oscillations at the fundamental cyclotron resonance in a gyrotron is the development of the previously proposed methods. According to calculations, it can confidently ensure selective generation at the second cyclotron harmonic in the $\mathrm{THz}$ gyrotron. The experimental verification of the proposed scheme should be implemented at the IAP in the near future. In principle, this method can be applied to ensure the generation selectivity at higher than the second cyclotron harmonics as well.

\section{Acknowledgements}

The work is supported by the Russian Science Foundation, Project \# 17-19-01605

\section{References}

1. A. V. Gaponov, M. I. Petelin, and V. K. Yulpatov, "The induced radiation of excited classical oscillators and its use in high-frequency electronics," Radiophys. Quantum Electron., vol. 10, no. 9, pp. 794$813,1967$.

2. G. S. Nusinovich, Introduction to the physics of gyrotrons. The Johns Hopkins University Press, Baltimore-London, 2004.

3. M. Yu. Glyavin, A. G. Luchinin, G. S. Nusinovich, J. Rodgers, D. G.Kashyn, C. A. Romero-Talamas, and R. Pu, "A $670 \mathrm{ghz}$ gyrotron with record power and efficiency," Appl. Phys. Lett., vol. 101, no. 15, p. $153503,2012$.

4. Yu. K. Kalynov, I. V. Osharin, and A. V. Savilov, "A method for suppression of spurious fundamental-harmonic waves in gyrotrons operating at the second cyclotron harmonic," Phys. Plasm., vol. 23, no. 5, p. $053116,2016$.

5. I. V. Bandurkin, Y. K. Kalynov, P. B. Makhalov, I. V. Osharin, A. V. Savilov, and I. V. Zheleznov, "Simulations of sectioned cavity for high-harmonic gyrotron," IEEE Trans. Electron Devices, vol. 64, no. 1, pp. 300-305, 2017.

6. G. S. Nusinovich and O. Dumbrajs, "Field formation in the interaction space of gyrotrons," J. Infrared Millimeter Terahertz Waves, vol. 37 , no. 1 , pp. 111-122, 2016.

7. G. G. Denisov, N. I. Zaitsev, S. V. Kuzikov, and M. E. Plotkin,"Enhancement of cavity selectivity in relativistic gyrotrons operated at axisymmetric modes," Radiophys. Quantum Electron., vol. 51, no. 10, pp. 756-767, 2008. 